\title{
Diagnóstico De Factores Conductuales Para El Trabajo En Equipo Con Estudiantes Universitarios
}

\author{
Erika Zavala López (MA) \\ Universidad Popular Autónoma del Estado de Puebla, México \\ David Flores Granados (PhD) \\ Universidad del Caribe, México \\ José Héctor Vicente Meneses Galván (PhD) \\ Irma Alejandra Hernández Vicente (PhD) \\ Universidad Popular Autónoma del Estado de Puebla, México
}

Doi:10.19044/esj.2018.v14n19p237 URL:http://dx.doi.org/10.19044/esj.2018.v14n19p237

\begin{abstract}
Teamwork is a temporary activity that takes place within organizations in order to perform sophisticated tasks. The behavior of the individuals that make up the team can positively or negatively affect the results obtained from them. Freud (1973) and later David McClelland (1989) attributed five (5) dimensions to human behavior: personality, values, culture, motivations, knowledge, and skills. Recent studies have deepened these aspects. This was done individually and based on different environments that relates to the field of higher education. The importance of teamwork has become prevalent in the world of work. This was despite the fact that this competence does not develop significantly in university education. This paper focuses on the development of a behavioral diagnostic instrument for teamwork based on ad hoc research and with the support of experts in organizational psychology, as well as the coherence of the instrument designed from the correlation of the items by categories of the five dimensions. This instrument was developed and validated to be reliable and applicable to university students. This was done in order to determine their behavioral profile which allows the designing strategies that helps to strengthen teamwork in classrooms.
\end{abstract}

Keywords: Teamwork, Personality, Values, Culture, Motivation

Resumen

El trabajo en equipo es una actividad temporal que se lleva a cabo a menudo dentro de las organizaciones para realizar tareas sofisticadas. Sin embargo, la conducta de los individuos que integran el equipo puede afectar positiva o negativamente los resultados obtenidos dentro de las mismas. Freud 
(1973) y posteriormente David McClelland (1989) atribuyeron a la conducta humana cinco dimensiones como son: personalidad, valores, cultura, motivaciones, conocimiento y habilidades. Estudios recientes han profundizado en estos aspectos pero de forma individual y en entornos distintos al ámbito de educación superior. La importancia del trabajo en equipo se ha vuelto imperante en el mundo laboral, a pesar de que esta competencia no se desarrolla significativamente en la formación universitaria. Dicho esto, en este artículo se describe la elaboración de un instrumento de diagnóstico conductual para el trabajo en equipo a partir de investigaciones ad hoc y con el respaldo de expertos en psicología organizacional, así como también la coherencia del instrumento diseñado a partir de la correlación de los ítems por categorías de las cinco dimensiones. Este instrumento fue elaborado y validado para ser fiable y aplicable a estudiantes universitarios con el fin de determinar su perfil conductual permitiendo diseñar estrategias que ayuden a fortalecer el trabajo en equipo en los salones de clases.

Palabras clave: Trabajo en equipo, Personalidad, Valores, Conocimiento, Cultura, Motivación, Universidad

\section{Introducción}

Entre las competencias sociales más demandadas en la vida laboral, de acuerdo con estudios realizados en el año 2012 por Millenial Branding Found, está el Trabajo en Equipo (Killonen, 2013). Se entiende como trabajo en equipo el conjunto de habilidades y conocimientos necesarios para realizar las tareas eficazmente, donde se requieren actitudes y conductas apropiadas por parte de cada uno de los miembros para lograr su funcionamiento (CannonBrowers et al., 1995). De acuerdo a las investigaciones, la parte conductual determina en gran medida el éxito en el trabajo en equipo.

En algunos de los primeros estudios dentro del área de psicología se propusieron modelos para explicar los mecanismos de cómo el individuo toma elementos del exterior para interiorizarlos y cómo estos influyen su comportamiento (Maslow, 1943; Skinner, 1953). Dichos modelos toman en cuenta las teorías del aprendizaje clásico y operante que hacen referencia al aprendizaje condicionado por los factores estimulantes del medio y por las propias experiencias, condicionando estos los comportamientos futuros.

Otras teorías como la del aprendizaje social recalcan la importancia de los modelos significativos como la familia, amigos, estereotipos o aspectos culturales, así como también la propia estructura cognitiva personal: las creencias, opiniones, actitudes y representaciones, las cuales inducen a los individuos a actuar de manera inconsciente formando esta estructura según sus experiencias positivas o negativas (Munuera, 2004). 
McCleland (1989), psicólogo estadounidense, retomó trabajos de varios de sus colegas para entender la conducta humana y los motivos que la generan, determinando que existen cinco principales factores que tenían influencia en ella: Conocimiento, Motivación, Valores, Personalidad y Cultura. Combinó variables como la Personalidad, la Intención del motivo, los Valores y las habilidades para predecir el comportamiento del ser humano.

El objetivo principal de este artículo es proponer y validar un instrumento que permita diagnosticar los principales factores conductuales que inciden en el trabajo en equipo en la etapa formativa universitaria.

\section{Metodología}

La metodología utilizada para la elaboración de este instrumento fue la siguiente (Figura 1).

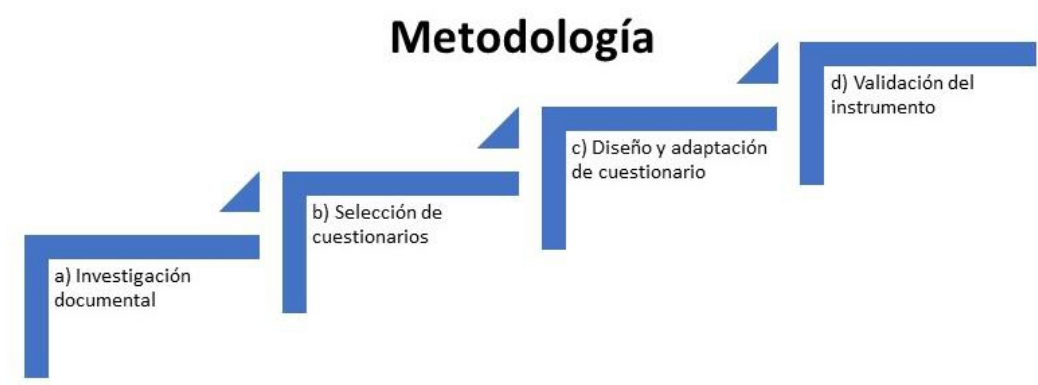

Figura 1. Metodología utilizada (Elaboración propia)

a) Una investigación documental que permita determinar los factores conductuales más importantes que influyen en el trabajo en equipo. Se buscó información en artículos y libros de las áreas organizacionales, psico-sociales y administrativas, así como bases de datos abiertas: Redalyc Scielo, Jurn y en Emerald Journals y EBSCOhost.

b) Selección de cuestionarios que describan los factores conductuales encontrados. Se revisaron una serie de instrumentos validados en los artículos seleccionados tomando en cuenta los factores a diagnosticar.

c) Diseño de cuestionario que contenga los factores encontrados. Se diseñó el cuestionario tomando en cuenta el punto anterior y adaptándolo a nuestra investigación al solicitar su revisión por parte de psicólogos organizacionales expertos en la materia. Se realizó una prueba piloto con 100 estudiantes universitarios para poder observar tiempos, claridad de conceptos y entendimiento de instrucciones en cada una de las secciones.

d) Validación del instrumento. Se validó el instrumento haciendo clasificaciones específicas de rasgos, graduaciones y validación 
estadística con el factor de correlación de ítems por categorías mediante $\alpha$ de Cronbach.

\section{Marco Teórico}

El trabajo en equipo dentro de las organizaciones es fundamental para poder lograr los objetivos y metas planteadas por las mismas. La conducta individual de los integrantes de un equipo dentro de una organización juega un papel muy importante para lograrlos. Existen cinco factores que pueden describir ampliamente la conducta individual de acuerdo con la psicología del comportamiento y la cognitiva: Conocimiento, Motivación, Valores, Cultura y Personalidad (McCleland, 1989). En las siguientes subsecciones se describe más ampliamente los factores mencionados y cómo se relacionan con la competencia de trabajo en equipo en las organizaciones.

\section{Conocimiento}

Desde la perspectiva de la psicología cognitiva, el estudio de la representación del conocimiento implica cómo las personas perciben los medios de su entorno; cómo aprenden, recuerdan, piensan y sienten en referencia a la información que reciben (Sternberg, 2011). Por otra parte, Nieto (2004) hace mención de que la dimensión cognitiva de las actitudes está basada en las creencias, percepciones, motivos personales y sociales y estos se vinculan con los afectos positivos y negativos que tienen las personas influyendo en sus actos.

McClelland (1989) tomó varias investigaciones de sus colegas como las de Freud donde comentaba que un elemento muy importante para el comportamiento del ser humano era "la comprensión de la situación por parte del individuo". De igual manera, enfatizó que para Heider una conducta se encuentra determinada por los esfuerzos y las habilidades de una persona en relación con una tarea, mientras que para Kintsch la fijación de objetivos facilita el rendimiento y el aprendizaje. Un referente obligatorio en las investigaciones conductuales de McClelland es el trabajo realizado por Skinner, en donde se señaló que los premios podían lograr conductas repetitivas. Otro elemento importante que retomó fue el planteado por Zajonc, en el que afirmó que la facilitación social mejora el rendimiento en las tareas simples, pero no en las complejas.

Por último, en la investigación de Viles (2013) se menciona que los modelos actuales de aprendizaje van dirigidos al desarrollo de competencias y actitudes desarrolladas en el proceso de aprendizaje, las cuales tienen que ser tutoradas por los profesores. En pocas palabras, el conocimiento explícito de los objetivos que deben cumplir los equipos de trabajo establece una base que proporciona mayores probabilidades de éxito en las tareas específicas. 


\section{Motivación}

Mateo (2001) define la motivación como "un proceso de surgimiento, mantenimiento y regulación de actos que producen cambios en el ambiente y que concuerdan con ciertas limitaciones internas (planes y programas)". Un punto en común de los estudios sobre motivación humana es que existen dos niveles de motivación: la motivación intrínseca y la motivación extrínseca. En la primera, el individuo actúa por sí mismo mientras que en la segunda actúa presionado o influenciado por el exterior, el medio ambiente que lo rodea. Deci (2006) desarrolla la teoría de Autodeterminación afirmando que esta motivación es autónoma y no necesita recompensas tangibles obligatorias. Toma también elementos de la motivación externa asegurando que se puede pasar de una motivación extrínseca a una intrínseca a través de un proceso controlado.

Por otra parte, Malone (1981) propone el desarrollo de la motivación intrínseca a través de ambientes que faciliten el aprendizaje y la convivencia como son: reto, fantasía y curiosidad. El desarrollo de las motivaciones también es descrito por Maslow (1943) en su Teoría de las Necesidades, donde afirma que el individuo pasa por diferentes etapas de manera ascendente iniciando con la Fisiológica, Seguridad, Afectiva/Afiliación, Estima y por último la Autorrealización. Keller (2000), por su parte, desarrolla su modelo ARCS (Atención, Relevancia, Confianza y Satisfacción) el cual representa las condiciones necesarias para que una persona esté completamente motivada. Un estudio extenso de los factores que incentivan la motivación se encuentra en el libro Motivación Humana del psicólogo David McClelland donde identifica tres motivos fundamentales: Logro, Poder y Afiliación (McClelland, 1989).

Los motivos de afiliación, logro y poder están implícitos en cada uno de los integrantes de un equipo de trabajo, por lo que es importante entender la ponderación de los motivos a nivel individual y general para establecer actividades que los conduzcan a la motivación intrínseca.

\section{Valores}

Los valores de las organizaciones permanecen a medida que sus integrantes se apropian de ellos, los mantienen o los fortalecen. Desde la perspectiva psicológica se definen a los valores como el motivo de la actividad. La formación de los valores a menudo pasa por tres etapas: reactiva, adaptativa y autónoma. En la etapa reactiva la exigencia externa, la presión, la amenaza de castigo o la recompensa de una promesa pueden ayudar al cumplimiento de un valor, sólo bajo la inmediata y directa presión externa. La etapa adaptativa sucede cuando el sujeto comprende que el cumplimiento del valor le permite obtener la recompensa o evitar el castigo, y es una vía aprendida y eficiente para lograr una recompensa y evitar un castigo. La etapa 
autónoma se logra cuando el sujeto elabora activamente la meta de cumplir con los valores independientemente de los premios y los castigos valores que están basados en las necesidades y metas propias que parten de él (González, 2002).

En el proceso de formación de los valores se deben de considerar ciertos factores primordiales como: el amor, la actitud incondicional, el afecto y comprensión hacia la persona a quien queremos inculcar un determinado valor y que la persona que desea inculcar el valor goce de prestigio en cuanto a cumplir con el valor de manera exitosa y satisfactoria. Así mismo, son también importantes la persuasión, la explicación del valor en sí mismo y de las ventajas y razones para cumplir con él y de esta manera lograr que el sujeto encuentre en el mismo la satisfacción de necesidades que ya actúan en él como la creatividad, la realización y la auto-realización (González, 2000).

Los valores se instauran a nivel psicológico de dos formas: valores formales que regulan el comportamiento de la persona ante situaciones de presión o control externo y los valores personalizados, que son expresiones legítimas y auténticas del sujeto que los asume (Durán, 2008). Los valores se trasmiten por medio del proceso de enseñanza-aprendizaje si se habla de la parte académica, y muchas veces a través del curriculum oculto, siendo el profesor un agente muy especial en la trasmisión de los mismos (Álvarez, 2007).

De acuerdo al modelo Axiológico de educación Integral se toma a la persona como: 1) Animal de inteligencia emocional (valores corporales, intelectuales, afectivos), 2) Singular y libre de sus decisiones (valores individuales, estéticos y morales), 3) De naturaleza abierta o relacional (Valores sociales, ecológicos, instrumentales, religiosos) (Gervilla, 2000).

\section{Cultura}

La cultura es el sistema de reglas y normas de inclusión y exclusión que en diferentes épocas permiten, a través de diferentes valores, aumentar las posibilidades de supervivencia y bienestar (Grubits \& Vera, 2009). Tomando el enfoque antropológico, se asume que las diferentes culturas valoran diferentes cosas, y lo que quiere cada individuo varía dependiendo de su cultura y lo que haya aprendido. La cultura determina los valores en la mayoría de los casos y estos se verán reflejados en la conducta del individuo (McClelland, 1989).

La cultura se presenta con diferentes niveles de estructuración a través de un proceso de formulación de reglas y normas que hacen que las personas se comporten de manera adecuada para que puedan tener una vida armónica en grupo. Los jóvenes están retados a moverse entre mundos múltiples como: conocimiento, cultura, conducta dentro de la propia familia, grupo de origen y escuela. Cada uno de estos mundos tienen diferentes creencias, valores, 
expectativas, acciones y respuestas emocionales, haciendo esta interacción un tanto difícil para los mismos (Vera \& Rodríguez, 2009). Los jóvenes necesitan asociar y/o interpretar de manera consciente o inconsciente significados comunes, permitiéndoles crear y mantener su propia cultura (Olmos \& Socha, 2006).

\section{Personalidad}

Sinisterra y Cruz (2009) definen la personalidad como las diferencias individuales que distinguen a un individuo de otro dentro del mismo grupo; todos sus rasgos y características determinarán su forma de comportarse, influyendo en este el ambiente social que modula y mantiene su comportamiento.

De acuerdo con la teoría fenomenológica de la personalidad, la personalidad es el resultado de motivaciones positivas que van evolucionando logrando en el individuo un mejor funcionamiento, y esta se construye según Rogers cuando sus acciones se dirigen a alcanzar sus metas (Morris \& Maisto, 1988). Aportando a esta teoría Allport afirmaba que los motivos de una persona determinan el nivel de madurez caracterizada por las actitudes, objetivos y valores de los individuos (Mischel, 1988), al igual que rasgos de la personalidad como el temperamento, la adaptabilidad, labilidad emocional y los valores.

Tomando la teoría de los rasgos de la personalidad, Engel (1996) define que la personalidad se refiere a las características particulares de cada individuo como el temperamento, la adaptabilidad, la labilidad emocional y los valores que le permiten al individuo girar en torno a una característica en particular, siendo estas características de la personalidad buenos predictores de la conducta y actuación de las personas (Morgeson, 2005).

\section{Métodos y materiales para la elaboración y validación de un diagnóstico conductual}

Un grupo piloto de 100 estudiantes de la Universidad del Caribe, específicamente de los Programas Educativos de Ingeniería de Datos e Inteligencia Organizacional, Telemática, Logística y Cadena de Suministros, fueutilizado para validar la correlación de las categorías en cada uno de los factores conductuales descritos previamente. El 18\% de los estudiantes fueron mujeres y el resto hombres en edades comprendidas entre 19 y 22 años, aplicando el instrumento en una sola sesión de forma electrónica a través de la plataforma Google form. La intención de la aplicación del instrumento fue primordialmente revisar la correlación de ítems por categoría de todos los factores a partir de la validación de los expertos en las áreas de diagnóstico y análisis posteriores. 
En este trabajo se planteó corroborar los valores reportados de las fuentes originales construyendo un único instrumento a partir de los 5 cuestionarios seleccionados, validando nuevamente así su factibilidad de uso en un instrumento compuesto y escalado.

\section{Elaboración del instrumento}

Una extensa revisión documental de diversos instrumentos que se han aplicado para cada uno de los cinco factores conductuales formaron la base de selección y modificación de nuestro instrumento, el cual está compuesto por 140 ítems en forma de preguntas para responder en escalas de tipo Likert diferenciadas, además de un conjunto de 150 palabras para la percepción de valores. El número de ítems también fue diferenciado de acuerdo a la ponderación de la significancia que los autores y expertos definieron.

En las siguientes subsecciones se muestra de forma detallada la composición de cada parte del instrumento.

\section{Elaboración del instrumento de diagnóstico para el factor de Personalidad}

Los cuestionarios de personalidad son los que tienen mayor referencia en el tiempo y han sido abordados por una gran cantidad de investigadores. En este instrumento en especial se hizo un cruce de los factores que propone Morgeson (2005), los cuales están enfocados en el trabajo en equipo y los 16 factores de la personalidad que propone Cattell (1989) en su herramienta para la contratación de personal.

El factor de la personalidad quedó plasmado en 84 preguntas y agrupado en 4 categorías (Ver Tabla 1 y Anexo A). El grupo piloto constó de 100 muestras; la validación estadística fue demostrada por Catell (2001) y revisada por un experto en psicología organizacional.

\begin{tabular}{|c|c|c|c|c|}
\hline Clasificación & $\begin{array}{c}\text { I } \\
\text { Agradabilidad }\end{array}$ & $\begin{array}{c}\text { II } \\
\text { Estabilidad } \\
\text { emocional }\end{array}$ & $\begin{array}{c}\text { III } \\
\text { Extroversión }\end{array}$ & $\begin{array}{c}\text { IV } \\
\text { Escrupulosidad/ } \\
\text { Conciencia }\end{array}$ \\
\hline Ítems & 1 al 23 & 24 al 47 & 48 al 64 & 65 al 84 \\
\hline Graduación & $\begin{array}{c}1 \text { al 3 } \\
\text { (Ponderación } \\
\text { diferenciada) }\end{array}$ & $\begin{array}{c}1 \text { al 3 } \\
\text { (Ponderación } \\
\text { diferenciada) }\end{array}$ & $\begin{array}{c}1 \text { al 3 } \\
\text { (Ponderación } \\
\text { diferenciada) }\end{array}$ & $\begin{array}{c}1 \text { al 3 } \\
\text { (Ponderación } \\
\text { diferenciada) }\end{array}$ \\
\hline
\end{tabular}

Tabla 1. Clasificación de los tipos de Personalidad diagnosticados por el instrumento.

\subsection{Elaboración del instrumento de diagnóstico para el factor de Motivación}

La personalidad y la motivación tienen un vínculo estrecho debido a que los propósitos tienen una vigencia que los conjuga. El test propuesto por McClelland (1989) y adaptado por Khurana y Joshi (2017) (ver Anexo B) sirvió como instrumento para diagnosticar tres tipos de motivación en 15 ítems 
con una graduación en escala de Likert de 5 niveles para cada uno (1=Completamente en desacuerdo, $2=$ En desacuerdo, $3=\mathrm{Ni}$ acuerdo ni desacuerdo, 4 = De acuerdo, 5 = Completamente de acuerdo). El grupo piloto constó de 100 estudiantes y la correlación de los ítems por clasificación se muestran en la Tabla 2 mediante la medida de correlación $\alpha$ de Cronbach.

\begin{tabular}{|c|c|c|c|}
\hline Clasificación & $\begin{array}{c}\text { I } \\
\text { Logro }\end{array}$ & $\begin{array}{c}\text { II } \\
\text { Poder }\end{array}$ & $\begin{array}{c}\text { III } \\
\text { Afiliación }\end{array}$ \\
\hline Ítems & 1 al 5 & 6 al 10 & 11 al 15 \\
\hline Graduación & $\begin{array}{c}1 \text { al 5 } \\
\text { (Escala } \\
\text { normalizada) }\end{array}$ & $\begin{array}{c}1 \text { al 5 } \\
\text { (Escala } \\
\text { normalizada) }\end{array}$ & $\begin{array}{c}1 \text { al 5 } \\
\text { (Escala } \\
\text { normalizada) }\end{array}$ \\
\hline $\begin{array}{c}\boldsymbol{\alpha} \text { de } \\
\text { Cronbach }\end{array}$ & 0.8630039851 & 0.7109908276 & 0.7395306135 \\
\hline
\end{tabular}

Tabla 2. Clasificación y factor de correlación $\alpha$ de Cronbach para la dimensión de Motivación.

\subsection{Elaboración del instrumento de diagnóstico para el factor de Valores}

Álvarez (2007) toma como modelo axiológico el cuestionario de respuestas valorativas de Cásares (1995) y lo enmarca en diez clasificaciones para obtener un instrumento de 250 palabras al que el aplicante responde en una escala de Likert estandarizada de 5 graduaciones ( $1=$ Muy Agradable, $2=$ Agradable, $3=$ Indiferente, $4=$ Desagradable, $5=$ Muy Desagradable). Tanto el cuestionario de Álvarez como el de Cásares constan de 250 palabras, 25 por cada categoría. En nuestro instrumento esta sección se re-escaló a 15 palabras por categoría (Ver Anexo C). El grupo piloto constó de 96 estudiantes y la correlación de los ítems por clasificación se muestran en la Tabla 3 mediante la medida de correlación $\alpha$ de Cronbach.

\begin{tabular}{|l|c|c|c|}
\hline $\begin{array}{c}\text { Clasificación de } \\
\text { valores }\end{array}$ & $\begin{array}{c}\text { No. De } \\
\text { Palabras }\end{array}$ & $\begin{array}{c}\alpha \text { de Cronbach } \\
\text { 150 palabras }\end{array}$ & $\begin{array}{c}\alpha \text { de Cronbach } \\
\text { 250 palabras }\end{array}$ \\
\hline I. Corporales & 1 a 15 & 0.8666922 & 0.8418633 \\
\hline II. Intelectuales & 16 a 30 & 0.8983729 & 0.915113 \\
\hline III. Afectivos & 31 a 45 & 0.9357494 & 0.9374306 \\
\hline IV. Estéticos & 46 a 60 & 0.9108845 & 0.9256175 \\
\hline V. Individuales & 61 a 75 & 0.9198733 & 0.9290377 \\
\hline VI. Morales & 76 a 90 & 0.9382099 & 0.9491015 \\
\hline VII: Sociales & 91 a 105 & 0.9149986 & 0.9197901 \\
\hline VIII. Ecológicos & 106 a 120 & 0.9606017 & 0.9544702 \\
\hline $\begin{array}{l}\text { IX. } \\
\text { Instrumentales }\end{array}$ & 121 a 135 & 0.8970207 & 0.9100635 \\
\hline X. Religiosos & 136 a 150 & 0.9755067 & 0.9748705 \\
\hline
\end{tabular}

Tabla 3. Clasificación y factor de correlación $\alpha$ de Cronbach para la dimensión de Valores.

El escalamiento para reducir el número de palabras en el instrumento propuesto se hizo con la selección de 15 palabras que maximizan el cálculo del factor de correlación por categoría; la comprobación de la validación en la re-escala se hizo mediante un análisis de regresión que se resume en la Tabla 
4 y en la Figura 2. Se puede observar que el escalamiento preserva el sentido del instrumento original con una pérdida despreciable.

\begin{tabular}{cr}
\hline \multicolumn{2}{c}{ Estadísticas de la regresión } \\
\hline Coeficiente de correlación múltiple & 0.93732271 \\
Coeficiente de determinación $\mathrm{R}^{\wedge} 2$ & 0.87857386 \\
$\mathrm{R}^{\wedge} 2$ ajustado & 0.86339559 \\
Error típico & 0.01313219 \\
Observaciones & 10 \\
\hline
\end{tabular}

Tabla 4. Estadísticos de Regresión para la comparación entre el instrumento de 250 palabras y el de 150.

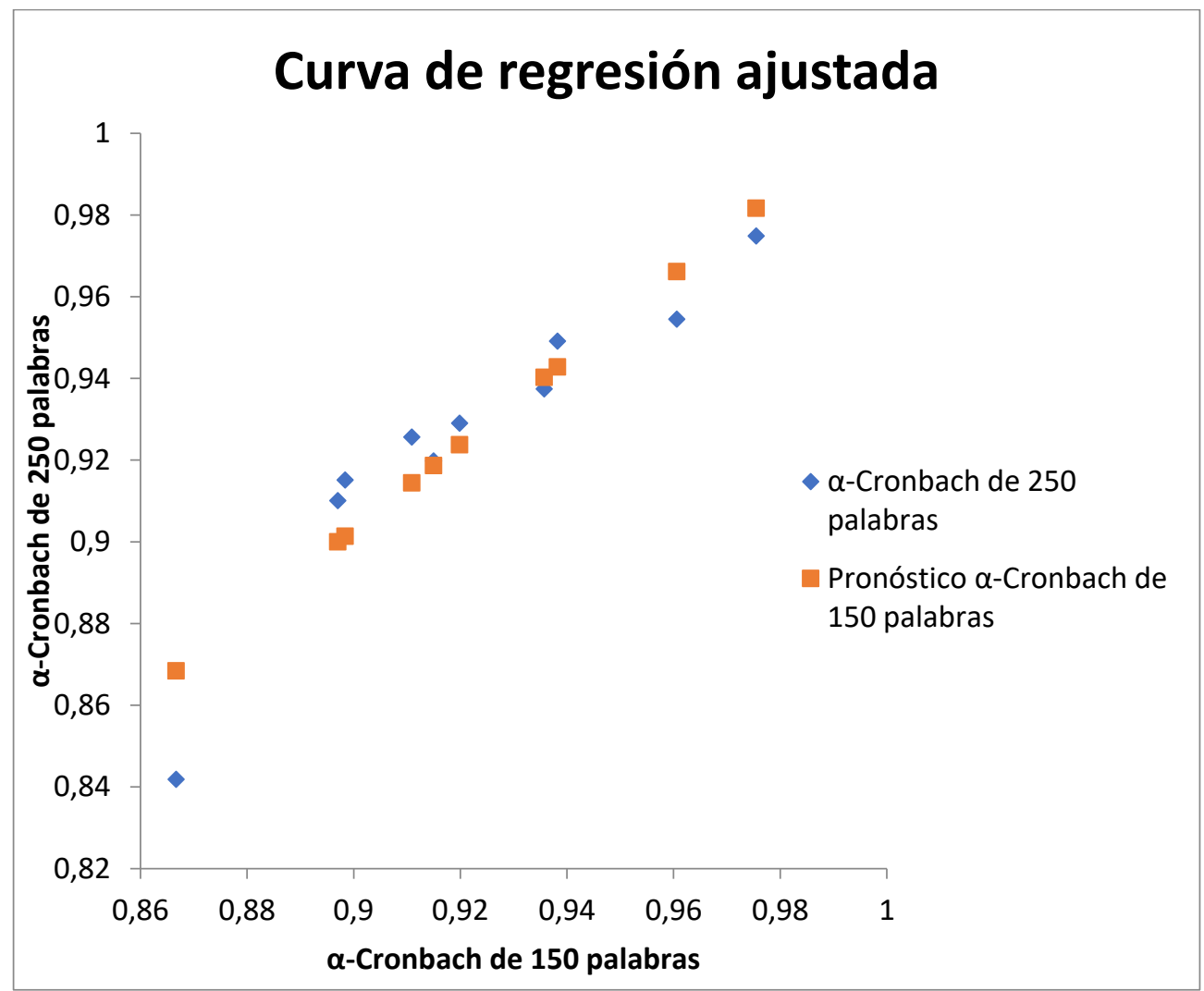

Figura 2. Curva de regresión ajustada para el escalamiento del instrumento de valores

Elaboración del instrumento de diagnóstico para el factor de Conocimientos y Habilidades

El trabajo en equipo requiere claridad en cuanto a los objetivos, tareas y productos a obtener en cada etapa de su desarrollo, y los conocimientos y habilidades de cada miembro contribuyen a que estos aspectos se logren con eficiencia en los tiempos asignados. Viles et al. (2013) propusieron un instrumento diagnóstico para identificar los conocimientos y habilidades 
principales asociados al trabajo en equipo en siete categorías (Ver Tabla 5 y Anexo D). Cada ítem tiene una graduación de 0 a $4(0=$ Totalmente en desacuerdo, 1 = En desacuerdo, 2 = Ni de acuerdo, ni en desacuerdo, $3=$ De acuerdo, 4 =Totalmente de acuerdo).

Puede notarse que la dispersión de clases implica una reducción de variables y, por lo tanto, una respuesta con valores bajos del coeficiente de Cronbach pero que se incrementa conforme se agrupan más variables. El grupo piloto constó de 96 estudiantes y la correlación de los ítems por clasificación se muestran en la tabla 5 mediante la medida de correlación $\alpha$ de Cronbach.

\begin{tabular}{|c|c|c|c|}
\hline Clasificación de Conocimientos & $\begin{array}{l}\text { No. de } \\
\text { Ítems }\end{array}$ & $\begin{array}{c}\alpha \text { de Cronbach } \\
\text { categoría }\end{array}$ & $\begin{array}{c}\alpha \text { de Cronbach } \\
\text { global }\end{array}$ \\
\hline $\begin{array}{l}\text { I. Participación y toma de } \\
\text { decisiones }\end{array}$ & 1 a 3 & 0.5960912052 & \multirow{7}{*}{0.9829300499} \\
\hline II. Gestión de conflictos & 4 a 5 & 0.29 & \\
\hline III. Resolución del problema & 6 a 7 & 0.4065769806 & \\
\hline IV. Comunicación interna & 8 a 10 & 0.4350839268 & \\
\hline V. Comunicación externa & 11 a 18 & 0.7260255158 & \\
\hline VI. Colaboración & 19 a 21 & 0.2608144796 & \\
\hline VII. Liderazgo & 22 a 23 & 0.4131191432 & \\
\hline
\end{tabular}

Tabla 5. Clasificación y factor de correlación $\alpha$ de Cronbach para la dimensión de Conocimientos y Valores.

\section{Elaboración del instrumento de diagnóstico para el factor de Cultura}

El instrumento que se adaptó para medir los factores culturales que más inciden en la organización para el trabajo en equipo fue el propuesto por Torres y Socha (2006) (Ver Anexo E). Las categorías se dividen en 6 aspectos que fueron avalados por ocho expertos. El grupo piloto constó de 102 estudiantes y la correlación de los ítems por clasificación se muestran en la Tabla 6 mediante la medida de correlación $\alpha$ de Cronbach.

\begin{tabular}{|c|c|c|c|}
\hline $\begin{array}{c}\text { Clasificación de } \\
\text { Cultura }\end{array}$ & Ítems & $\begin{array}{c}\alpha \text { de Cronbach } \\
\text { categoría }\end{array}$ & $\begin{array}{c}\alpha \text { de Cronbach } \\
\text { global }\end{array}$ \\
\hline I. Valores & 1 a 3 & 0.7698745639 & \multirow{6}{*}{0.8676266918} \\
\hline II. Creencias & 4 a 6 & 0.2916866983 & \\
\hline III. Clima & 7 a 9 & 0.777438269 & \\
\hline IV. Normas & 10 a 12 & 0.3891815474 & \\
\hline V. Símbolos & 13 a 15 & 0.4413955732 & \\
\hline VI. Filosofía & 16 a 18 & 0.8371051391 & \\
\hline
\end{tabular}

Tabla 6. Clasificación y factor de correlación $\alpha$ de Cronbach para la dimensión de Cultura Organizacional.

\section{Conclusión}

Con este instrumento se logró conjuntar las variables principales que intervienen en la conducta de los individuos y que influyen fuertemente en su comportamiento grupal, validando así cada una de las dimensiones señaladas 
nuevamente: Conocimiento, Motivación, Valores, Cultura y Personalidad. Para la sección de Personalidad el instrumento fue validado previamente por Cattell (2001), en la parte de Motivación se logró un $\alpha$ de Cronbach superior al .70 teniendo así la confiabilidad de esta sección.

Para la dimensión de Valores se escaló el instrumento original que inicialmente era de 250 palabras para reducirlo a 150 , conservando el sentido del instrumento con un error típico de 0.01313219. En el factor de Conocimiento se notó que en la dispersión de clases existió un bajo coeficiente de Cronbach, pero en su conjunto se obtuvo un .9829300499 obteniendo así su validación. Por último, en la sección de Cultura se logró una correlación de .8676266918 .

Como se puede apreciar, este instrumento reconstruido cumple con la validación estadística y evaluativa por experto en el área del comportamiento, necesarias para realizar un diagnóstico muy cercano a la realidad en cuanto a la Personalidad, Valores, Cultura, Motivación, Conocimiento y Habilidades, factores involucrados en el trabajo en equipo. Al evaluar el comportamiento grupal de estudiantes universitarios, y ayudar ayudando a diseñar estrategias específicas para cada grupo se permite fortalecer el trabajo en equipo en las aulas de clase.

También puede ser utilizado para diagnosticar el perfil de los maestros, conociendo si estos saben, quieren o se sienten cómodos al promover en sus aulas estrategias para desarrollar el trabajo en equipo. Igualmente, se puede utilizar el instrumento para diagnosticar a los aspirantes de un empleo para conocer su perfil específico en los factores que facilitarán el trabajo en equipo dentro de la organización.

\section{References:}

1. Álvarez, J. (2007). Test Axiológico. Un instrumento para detectar valores. Revista Portuguesa de pedagogía.

2. Cannon-Bowers, J. A., Tannenbaum, S. I., Salas, E., \& Volpe, C. E. (1995). Defining team competencies and establishing team training requirements. In R. Guzzo, E. Salas, \& Associates (Eds.), Team effectiveness and decision making in organizations (pp. 333-380). San Francisco, CA: Jossey-Bass

3. Casares, P. (1995). Test de valores: un instrumento para la evaluación. . Revista Española de Pedagogía, 513-537.

4. Cattell, R \& Sierra, G. (2001). Cuestionario de 16 factores de la personalidad (16FP). México: Editorial El Manual Moderno.

5. Deci, E., \& Gagné, M. (2006). Self-determination theory and work motivation. Journal of Organizational Behavior, 331-362.

6. Durán (2008). La administracion por valores: una metodología humanista de cambio cultural en la empresa. Ciencias Economicas. 
7. Engel, B. (1996). Teorías de la personalidad. México: Mc Graw Hill.

8. Freud.S. (1973). La interpretación de los sueños. Madrid: Biblioteca Nueva.

9. Gervilla, E. (2000). Un modelo axiológico de educación integral. Revista Española de Pedagogía, 215-395.

10. Gonzalez Martin, M. d. (2008). Trabajo en equipo y revisiones cruzadas para convertir al alumno en protagonista de su proceso de aprendizaje. Universidad Salamanca España.

11. Gonzalez, D. (2000). Los valores y su formación : una interpretación psicológica. Revista Cubna de Psicología.

12. Grubits, S., \& Vera, C. (2009). La psicología Social y el concepto de Cultura. Psicología \& Sociedade, 100-107.

13. Keller, J. (2000). How to integrate learner motivation planning into lesson planning: The ARCS model Approach. VII Seminario, Santiago Cuba, 1-13.

14. Khurana, H., \& Joshi, V. (2017). Motivation and its Impact on Individual Performance: a Comparative Study Based on McClelland's Three Need Model. Clear International Journal of Research in Commerce \& Management, 110-116.

15. Kyllonen, P. (2013). The importance of Soft Skills. Changemang.org.

16. Malone, T. (1981). Toward a Theory of Intrinsically Instruction. Cognitive Science, 333-369.

17. Maslow, A. (1943). A theory of Human Motivation. Phycological Review, 370-396.

18. Mateo, M. (2001). La motivación: pilar básico de todo tipo de esfuerzo. Revista de Relaciones Laborales, 7-21

19. McClelland, D. (1989). Estudio de la Motivación Humana. Madrid: Narcea, SA. de Ediciones .

20. Mischel, W. (1988). Teorías de la personalidad. México: Mc Graw Hill.

21. Morgeson, F. (2005). Selecting individuals in team settings:The importance of social skills, personality characteristics and teamwork knowledge. Personnel Psychology, 583-611.

22. Morris, G., \& Maisto, A. (1988). Psicología . México: Prentice Hall.

23. Munuera, J. (2004). Dimensión Social. Psicología para ciencias de la salud: estudio del comportamiento humano ante la enfermedad.

24. Nieto, J. (2004). Dimensión social. Psicología para ciencias de la salud: estudio del comportamiento humano ante la enferedad.

25. Olmos, M., \& Socha, K. (2006). Diseño y validación mediante jueces expertos del instrumento para evaluar cultura organizacional. Universidad de la Sabana. 
26. Sinisterra, M., \& Cruz, P. (2009). Teorías de la personalidad: Un análisishistórico del concepto y su medicion. Psychologia. Avances de la disciplina, 81-107.

27. Sternberg, K. (2011). Cognitive Psychology. Belmont: Cengage Learning.

28. Vera, J., \& Rodríguez, C. (2009). La Psicología Social y el Concepto de Cultura. Centro de Investigación en alimentación y desarrollo.

29. Viles, E., Zárraga, M. \& Jaca, C. (2013). Herramienta para evaluar el funcionamiento de los equipos de trabajo en entornos docentes. Tangible Capital.

\section{Anexos}

ANEXO A. Ítems de la dimensión Personalidad

\begin{tabular}{|c|c|}
\hline Número & Pregunta \\
\hline 01 & $\begin{array}{l}\text { Yo prefiero la música semiclásica a las canciones populares (Cierto, Intermedio, } \\
\text { Falso) }\end{array}$ \\
\hline 02 & $\begin{array}{l}\text { Casi siempre puedo tolerar a la gente vanidosa que se cree la gran cosa (Sí, En duda, } \\
\text { No) }\end{array}$ \\
\hline 03 & $\begin{array}{c}\text { La mayoría de la gente que conozco me considera como un conversador agradable } \\
\text { (Sí, Indeciso, No) }\end{array}$ \\
\hline 04 & $\begin{array}{c}\text { Cuando estoy encargado de hacer algo, yo insisto en que se sigan mis instrucciones } \\
\text { o bien renuncio. (Sí, A veces, No) }\end{array}$ \\
\hline 05 & $\begin{array}{c}\text { Me gusta asistir a espectáculos, o ir a fiestas (Más de una vez a la semana, Una vez a } \\
\text { la semana, Menos de una vez a la semana) }\end{array}$ \\
\hline 06 & Si alguien se enoja conmigo, yo: (Trato de calmarlo, Indeciso, Me irrito) \\
\hline 07 & $\begin{array}{l}\text { Cuando leo un artículo injusto en una revista tiendo a olvidarlo, más que a sentir } \\
\text { ganas de "devolverles el golpe" (Cierto, Dudoso, Falso) }\end{array}$ \\
\hline 08 & $\begin{array}{l}\text { Yo tengo indudablemente menos amigos que la mayoría de la gente (Sí, En duda, } \\
\text { No) }\end{array}$ \\
\hline 09 & $\begin{array}{c}\text { Detestaría estar en un lugar donde no hubiera muchas personas con quien platicar } \\
\text { (Cierto, Indeciso, Falso) }\end{array}$ \\
\hline 10 & $\begin{array}{c}\text { Cuando la gente mandona trata de imponerse, yo hago exactamente lo contrario de } \\
\text { lo que ellas quieren (Cierto, Intermedio, Falso). }\end{array}$ \\
\hline 11 & $\begin{array}{c}\text { Es una regla que mis jefes o los miembros de mi familia me consideren culpable } \\
\text { sólo si existe una razón real (Cierto, Intermedio, Falso). }\end{array}$ \\
\hline 12 & $\begin{array}{l}\text { Asisto a reuniones sociales sólo cuando tengo que hacerlo, de otra manera trato de } \\
\text { evitarlas (Sí, Indeciso, No) }\end{array}$ \\
\hline 13 & $\begin{array}{l}\text { Ser precavido y esperar poco es mejor que ser optimista y esperar siempre el éxito } \\
\text { (Cierto, Indeciso, Falso) }\end{array}$ \\
\hline 14 & $\begin{array}{c}\text { Si estoy completamente seguro de que una persona es injusta o es egoísta, se lo digo, } \\
\text { aunque me traiga problemas gente (Sí, Intermedio, No) }\end{array}$ \\
\hline 15 & $\begin{array}{c}\text { A veces yo hago observaciones tontas en broma, solo para que las personas se } \\
\text { sorprendan y ver qué es lo que dicen (Sí, Intermedio, No) }\end{array}$ \\
\hline 16 & $\begin{array}{c}\text { Yo paso mucho de mi tiempo libre platicando con amigos sobre reuniones sociales } \\
\text { en las que nos divertimos en el pasado (Sí, Intermedio, No) }\end{array}$ \\
\hline 17 & $\begin{array}{l}\text { Me agrada hacer cosas temerarias y atrevidas nada más por gusto (Sí, Intermedio, } \\
\text { No) }\end{array}$ \\
\hline 18 & $\begin{array}{l}\text { Si nadie se da cuenta de una buena observación mía (No le doy importancia, } \\
\text { indeciso, Repito la frase para que la gente pueda escucharla nuevamente) }\end{array}$ \\
\hline 19 & $\begin{array}{l}\text { Preferiría vestirme sencilla y correctamente, y no con un estilo peculiar y llamativo. } \\
\text { (Cierto, Intermedio, Falso) }\end{array}$ \\
\hline
\end{tabular}




\begin{tabular}{|c|c|}
\hline 20 & $\begin{array}{l}\text { Me llama más la atención pensar una tarde con un pasatiempo tranquilo que en una } \\
\text { fiesta animada (Cierto, Intermedio, Falso). }\end{array}$ \\
\hline 21 & $\begin{array}{c}\text { A veces me causa problemas el que la gente hable mal de mí a mis espaldas, sin } \\
\text { tener razón (Sí, Indeciso, No) }\end{array}$ \\
\hline 22 & Me consideran como una persona muy entusiasta (Sí, Intermedio, No) \\
\hline 23 & $\begin{array}{l}\text { Prefiero un trabajo con variedad, viajes y cambios, aunque tenga riesgos. (Sí, } \\
\text { Intermedio, No) }\end{array}$ \\
\hline 24 & $\begin{array}{c}\text { Yo tengo la energía suficiente para enfrentarme a mis dificultades. (Siempre, } \\
\text { Generalmente, Pocas veces) }\end{array}$ \\
\hline 25 & $\begin{array}{c}\text { Me siento un poco nervioso ante los animales salvajes, aunque estén enjaulados. (Sí, } \\
\text { Indeciso, No) }\end{array}$ \\
\hline 26 & $\begin{array}{c}\text { En ocasiones tengo la sensación de un vago peligro o un miedo súbito por razones } \\
\text { que no comprendo. (Sí, Intermedio, No) }\end{array}$ \\
\hline 27 & $\begin{array}{l}\text { Cuando me regañan por algo que no hice, no me siento culpable. (Cierto, } \\
\text { Intermedio, No) }\end{array}$ \\
\hline 28 & $\begin{array}{c}\text { A veces no puedo dormir porque una idea me da vueltas en la cabeza (Cierto, } \\
\text { Dudoso, Falso) }\end{array}$ \\
\hline 29 & $\begin{array}{l}\text { En mi vida privada, casi siempre alcanzo las metas que me propongo. (Cierto, } \\
\text { Dudoso, Falso) }\end{array}$ \\
\hline 30 & $\begin{array}{l}\text { Me siento muy afligido cuando la gente me critica en público. (Cierto, Intermedio, } \\
\text { Falso) }\end{array}$ \\
\hline 31 & $\begin{array}{c}\text { Cuando el jefe (o el maestro) me llama: (Veo una oportunidad para hablar de cosas } \\
\text { que me interesan, Indeciso, Temo haber hecho algo mal) }\end{array}$ \\
\hline 32 & $\begin{array}{l}\text { Yo tengo algunas cualidades, por lo que me siento superior a la mayoría de la gente. } \\
\text { (Sí, Dudoso, No) }\end{array}$ \\
\hline 33 & $\begin{array}{c}\text { Algunas veces no tengo ganas de ver a nadie. (Raras veces, Intermedio, Muy } \\
\text { frecuentemente) }\end{array}$ \\
\hline 34 & $\begin{array}{l}\text { A veces las personas me dicen que muestro de manera demasiado clara mi agitación. } \\
\text { (Sí, Intermedio, No) }\end{array}$ \\
\hline 35 & $\begin{array}{l}\text { Yo no sé por qué, pero algunas personas como que me ignoran o me evitan. (Cierto, } \\
\text { Indeciso, Falso) }\end{array}$ \\
\hline 36 & $\begin{array}{c}\text { Las personas me tratan con menos consideración de lo que merecen mis buenas } \\
\text { intenciones (A menudo, En ocasiones, Nunca) }\end{array}$ \\
\hline 37 & $\begin{array}{c}\text { Las personas dicen que soy descuidado a veces, aunque ellas me consideren } \\
\text { simpático. (Sí, Intermedio, No) }\end{array}$ \\
\hline 38 & Cuando la gente no es razonable (Me quedo callado, Intermedio, Los desprecio) \\
\hline 39 & $\begin{array}{l}\text { Si alguien habla en voz alta cuando estoy escuchando música puedo (Concentrarme } \\
\text { en la música y no me molesta, Intermedio, Acaban con mi placer y me molesto) }\end{array}$ \\
\hline 40 & $\begin{array}{l}\text { Yo siento que me van a castigar, aun cuando no haya hecho nada malo. (A menudo, } \\
\text { Ocasionalmente, Nunca) }\end{array}$ \\
\hline 41 & $\begin{array}{c}\text { La idea de que las enfermedades tienen causas tanto físicas como mentales es muy } \\
\text { exagerada. (Sí, Intermedio, No) }\end{array}$ \\
\hline 42 & $\begin{array}{c}\text { Yo puedo trabajar con cuidado en muchas cosas sin ser molestado por las personas } \\
\text { que hacen ruido a mí alrededor. (Sí, Intermedio, No) }\end{array}$ \\
\hline 43 & $\begin{array}{l}\text { Me consideran acertadamente como una persona trabajadora y de mediano éxito. (Sí, } \\
\text { Indeciso, No) }\end{array}$ \\
\hline
\end{tabular}

\begin{tabular}{|c|c|}
\hline 44 & $\begin{array}{c}\text { Si las personas abusan de mi amistad, no lo resiento y lo olvido pronto. (Cierto, } \\
\text { Indeciso, Falso) }\end{array}$ \\
\hline 45 & Tengo sueños tan intensos que me inquietan cuando duermo. (A menudo, \\
Ocasionalmente, Casi nunca)
\end{tabular}




\begin{tabular}{|c|c|}
\hline 48 & En las reuniones sociales (me hago notar, no sé, prefiero permanecer a distancia) \\
\hline 49 & $\begin{array}{l}\text { Si yo tuviera el mismo sueldo y horario me gustaría más trabajar como: (carpintero o } \\
\text { cocinero, indeciso, mesero en un buen restaurante) }\end{array}$ \\
\hline 50 & Yo soy apto para algunos. (Pocos empleos, varios empleos, muchos empleos) \\
\hline 51 & $\begin{array}{l}\text { Siempre me gusta participar en reuniones concurridas, por ejemplo: una fiesta, un } \\
\text { mitin. (Sí, Intermedio, No) }\end{array}$ \\
\hline 52 & $\begin{array}{c}\text { En santos y cumpleaños. (Me gusta hacer regalos personales, Indeciso, Creo que es } \\
\text { un poco molesto comprar regalos) }\end{array}$ \\
\hline 53 & $\begin{array}{c}\text { Al inventar algo útil, preferiría: (Perfeccionarlo en el laboratorio, Indeciso, } \\
\text { Vendérselo a la gente) }\end{array}$ \\
\hline 54 & $\begin{array}{c}\text { Cuando estoy en un grupo pequeño, me agrada permanecer en silencio y mejor dejar } \\
\text { que otros hablen. (Cierto, Intermedio, Falso) }\end{array}$ \\
\hline 55 & $\begin{array}{c}\text { Sería muy interesante trabajar en una empresa (Hablando con los clientes, } \\
\text { Intermedio, Llevando las cuentas y el archivo) }\end{array}$ \\
\hline 56 & $\begin{array}{c}\text { Para mí, es fácil incorporarme con las personas en una reunión social. (Cierto, } \\
\text { Intermedio, Falso) }\end{array}$ \\
\hline 57 & $\begin{array}{c}\text { Cuando un poco de diplomacia y persuasión son necesarias para que la gente actúe, } \\
\text { yo generalmente soy el primero en fomentarlas. (Cierto, Indeciso, Falso) }\end{array}$ \\
\hline 58 & $\begin{array}{l}\text { Si los salarios fueran los mismos preferiría ser: (Abogado, Indeciso, Navegante o } \\
\text { Piloto) }\end{array}$ \\
\hline 59 & $\begin{array}{l}\text { Me considero una persona muy sociable con la que es fácil llevarse. (Sí, Intermedio, } \\
\text { No) }\end{array}$ \\
\hline 60 & $\begin{array}{c}\text { En mi trato social: (demuestro mis emociones como quiero, intermedio, } \\
\text { me guardo mis emociones) }\end{array}$ \\
\hline 61 & Preferiría la vida de: (Un artista, indeciso, Secretario de un club social) \\
\hline 62 & Me disgusta un poco que un grupo me observe cuando trabajo. (Sí, Intermedio, No) \\
\hline 63 & $\begin{array}{c}\text { Si me pidieran que trabajara en una obra de caridad: (Aceptaría, Indeciso, } \\
\text { Diría con cortesía que estoy ocupado) }\end{array}$ \\
\hline 64 & Me considero un tipo enérgico que se mantiene activo. (Sí, Indeciso, No) \\
\hline 65 & $\begin{array}{l}\text { Cuando un hombre es deshonesto, casi siempre lo puedes notar en su cara. (Sí, En } \\
\text { duda, No) }\end{array}$ \\
\hline
\end{tabular}

\begin{tabular}{|c|c|}
\hline 66 & $\begin{array}{c}\text { Yo hablo sobre mis sentimientos. (Sólo si es necesario, Intermedio, Cada vez que } \\
\text { tengo la oportunidad) }\end{array}$ \\
\hline 67 & $\begin{array}{c}\text { Cuando veo a personas desaliñadas y desaseadas yo: (Las acepto, Intermedio, Me } \\
\text { disgusto) }\end{array}$ \\
\hline 68 & $\begin{array}{c}\text { Para los padres es más importante: (Ayudar a sus niños a desarrollar sus afectos, } \\
\text { Intermedio, Enseñar a sus niños cómo controlar sus emociones) }\end{array}$ \\
\hline 69 & $\begin{array}{c}\text { En una tarea de grupo, yo más bien trataría de: (Imponer acuerdos, Intermedio, } \\
\text { Hacer apuntes y ver que se obedezcan las reglas) }\end{array}$ \\
\hline 70 & $\begin{array}{c}\text { De vez en cuando siento la necesidad de realizar actividades físicas rudas o pesadas. } \\
\text { (Sí, Indeciso, No) }\end{array}$ \\
\hline 71 & $\begin{array}{c}\text { Preferiría juntarme con gente bien educada, a juntarme con individuos toscos y } \\
\text { rebeldes. (Sí, Intermedio, No) }\end{array}$ \\
\hline 72 & $\begin{array}{c}\text { Yo como mis alimentos con placer, aunque no siempre tan cuidadosa y } \\
\text { apropiadamente como algunas personas. (Cierto, Intermedio, Falso) }\end{array}$ \\
\hline 73 & $\begin{array}{c}\text { En una situación que puede volverse peligrosa yo creo conveniente hacer ruido y } \\
\text { escándalo, aunque se pierda la serenidad y la cortesía. (Sí, Indeciso, No) }\end{array}$ \\
\hline 74 & $\begin{array}{c}\text { Si alguien me dice algo, que sé que es falso, yo muy probablemente me diga: (él es } \\
\text { un embustero, intermedio, aparentemente él está mal informado) }\end{array}$ \\
\hline 75 & La escena de un cuarto desarreglado me molesta. (Sí, Intermedio, No) \\
\hline 76 & $\begin{array}{c}\text { Me gustaría trabajar como vigilante con criminales que estuvieran en libertad bajo } \\
\text { palabra. (Sí, Intermedio, No) }\end{array}$ \\
\hline
\end{tabular}




\begin{tabular}{|c|c|}
\hline 77 & $\begin{array}{l}\text { Uno debe de tener cuidado al mezclarse con toda clase de extraños, por el peligro de } \\
\text { una infección. (Sí, Intermedio, No) }\end{array}$ \\
\hline 78 & $\begin{array}{c}\text { En un viaje al extranjero, yo preferiría ir en un tour planeado con un conductor de } \\
\text { viaje experimentado, que planear por mí mismo los lugares que desearía visitar. (Sí, } \\
\text { Indeciso, No) }\end{array}$ \\
\hline 79 & $\begin{array}{l}\text { Siempre mi criterio para cualquier decisión se basa en los principios del bien y del } \\
\text { mal. (Sí, Indeciso, No) }\end{array}$ \\
\hline 80 & Algunas cosas me enojan tanto que prefiero no hablar de ellas. (Sí, Intermedio, No) \\
\hline 81 & $\begin{array}{l}\text { Es muy importante en la educación: (Dar suficiente afecto a los niños, } \\
\text { Intermedio, Que los niños aprendan hábitos y actitudes convenientes) }\end{array}$ \\
\hline 82 & $\begin{array}{c}\text { Soy una persona bastante estricta que insiste siempre en hacer las cosas tan } \\
\text { correctamente como sea posible. (Cierto, Intermedio, Falso) }\end{array}$ \\
\hline 83 & $\begin{array}{c}\text { Me agradan los trabajos que requieren concentración y habilidades precisas. (Sí, } \\
\text { Indeciso, No) }\end{array}$ \\
\hline 84 & $\begin{array}{l}\text { Si yo viera pelear a los niños de mi vecino. (Dejaría que se arreglaran solos, No } \\
\text { sabría qué hacer, Prefiero permanecer a distancia) }\end{array}$ \\
\hline
\end{tabular}

Tabla de Ítems para el instrumento consultivo de Personalidad.

\section{ANEXO B. Ítems de la dimensión Motivación}

\begin{tabular}{|c|c|}
\hline Número & Pregunta \\
\hline 01 & Trato firmemente de mejorar mi rendimiento académico \\
\hline 02 & Disfruto de un desafío difícil \\
\hline 03 & Quiero saber cómo estoy progresando a medida que completo las tareas \\
\hline 04 & Me gusta establecer y lograr metas realistas \\
\hline 05 & Disfruto con satisfacción, haber completado una tarea difícil \\
\hline 06 & Me gusta competir y ganar \\
\hline 07 & Me gusta estar a cargo de situaciones o actividades \\
\hline 08 & Yo enfrento a las personas que hacen cosas con las que no estoy de acuerdo \\
\hline 09 & Me gusta influenciar a otros para que hagan lo que yo quiero \\
\hline 10 & A menudo trabajo para tener más control sobre los eventos que me rodean \\
\hline 11 & A menudo me encuentro hablando con quienes me rodean sobre cuestiones \\
& no relacionadas con la universidad \\
\hline 12 & Quiero ser querido(a) por los demás \\
\hline 13 & Tiendo a construir relaciones cercanas con compañeros de la universidad \\
\hline 14 & Me gusta pertenecer a grupos y organizaciones \\
\hline 15 & Me gusta trabajar con otros más que trabajar solo \\
\hline
\end{tabular}

\section{ANEXO C. Ítems de la dimensión Valores}

Palabras para valores Corporales: Alimentarse, Asearse, Comer, Cuerpo, Descanso, Ducharse, Higiene, Lavarse, Masaje, Placer, Peinarse, Salud, Sexualidad, Siesta, Vitalidad.

Palabras para valores Intelectuales: Aprender, Asignatura, Biblioteca, Ciencia, Conferencia, Conocimiento, Deducir, Facultad, Investigación, Pensar, Profesor, Reflexionar, Saber, Tema Universidad.

Palabras para valores Afectivos: Abrazar, Acariciar, Afectividad, Amar, Besar, Cariño, Caricia, Enamorarse, Esposos, Familia, Hijos, Novio/Novia, Sentimientos, Ser Amado, Ternura. 
Palabras para valores Estéticos: Arte, Arquitectura, Cantar, Dibujo, Elegancia, Escultura, Estética, Fotografía, Hermosura, Literatura, Moda, Pintura, Poesía, Recital, Teatro.

Palabras para valores Individuales: Auténtico, Autocontrol, Autonomía, Autosuperación, Carácter, Conciencia, Experiencia, Identidad, Independencia, Interioridad, Intimidad, Libertad Original, Personalidad, Valentía.

Palabras para valores Morales: Ayudar, Bien, Dignidad, Esfuerzo, Ética, Fortaleza, Generosidad, Honradez, Igualdad, Paz, Perseverancia, Respeto, Sinceridad, Solidaridad, Voluntad.

Palabras para valores Sociales: Asociación, Ciudadano, Convivencia, Colaborar, Comunidad, Constitución, Consenso, Diálogo, Estado, Igualdad de Oportunidades, Ley, Manos Unidas, Multiculturalidad, Pacifismo, Tolerancia. Palabras para valores Ecológicos: Biodegradable, Bosque, Campo, Desarrollo, Sostenido, Ecologismo, Excursión, Flores, Jardín, Lago, Lluvia, Montaña, Nieve, Parque Natural, Río, Vegetación.

Palabras para valores Instrumentales: Ahorrar, Avión, Coche, Dinero, Electricidad, Herencia Industria, Internet, Millonario, Celular, Riqueza, Ropa, Sueldo, Video, Vivienda.

Palabras para valores Religiosos: Bautismo, Bendecir, Biblia, Catequesis, Clases de Religión, Creyente, Cuaresma, Dios, Evangelio, Iglesia, Jesucristo, Misa.

\begin{tabular}{|c|c|}
\hline \multicolumn{2}{|c|}{ ANEXO D. Ítems de la dimensión Conocimientos y Habilidades } \\
\hline Número & Ítem \\
\hline 01 & En tus clases se han establecido normas internas que facilitaron el trabajo en equipo \\
\hline 02 & He participado en las tareas de equipo \\
\hline 03 & $\begin{array}{l}\text { Las decisiones en el equipo se han tomado teniendo en cuenta la opinión de todos los } \\
\text { miembros }\end{array}$ \\
\hline 04 & $\begin{array}{c}\text { Las discrepancias en el equipo me han permitido considerar nuevas ideas o nuevos } \\
\text { puntos de vista }\end{array}$ \\
\hline 05 & $\begin{array}{l}\text { No ha habido conflictos de tipo interpersonal (puntuar con 0) y si los ha habido, se } \\
\text { han resuelto sin que nadie se haya sentido perjudicado }\end{array}$ \\
\hline 06 & $\begin{array}{c}\text { Se han utilizado los datos y un método para la resolución de ejercicios o problemas } \\
\text { planteados }\end{array}$ \\
\hline 07 & $\begin{array}{c}\text { Se ha potenciado la creatividad para la resolución de los problemas o propuestas } \\
\text { planteadas }\end{array}$ \\
\hline 08 & El trabajo del equipo ha transcurrido en un ambiente de confianza \\
\hline 09 & $\begin{array}{c}\text { A pesar de las diferencias entre los miembros del equipo, ha existido un ambiente de } \\
\text { respeto sobre todos }\end{array}$ \\
\hline 10 & En general, la comunicación ha sido buena entre los miembros del equipo \\
\hline 11 & Los objetivos se han trasmitido bien al equipo y han sido comprendidos \\
\hline 12 & $\begin{array}{l}\text { El profesor ha facilitado los recursos (información, materiales, tiempo o de otro tipo) } \\
\text { que el equipo ha necesitado }\end{array}$ \\
\hline 13 & $\begin{array}{c}\text { Las tareas y actividades concretas a realizar por el equipo han estado claras tanto en } \\
\text { contenido como en plazo (tiempo de realización) }\end{array}$ \\
\hline
\end{tabular}




\begin{tabular}{|c|c|}
\hline 14 & $\begin{array}{c}\text { Los criterios de evaluación establecidos por los maestros se han transmitido bien al } \\
\text { equipo y han sido comprendidos al inicio del trabajo }\end{array}$ \\
\hline 15 & $\begin{array}{l}\text { Cuando hemos formado equipos académicos hemos tenido acceso a la información } \\
\text { necesaria para trabajar }\end{array}$ \\
\hline 16 & $\begin{array}{l}\text { En caso de necesidad, los equipos en los que he participado se han podido comunicar } \\
\text { fácilmente con el profesor de las asignaturas involucradas }\end{array}$ \\
\hline 17 & $\begin{array}{l}\text { Los equipos en donde he participado hemos recibido información acerca del } \\
\text { resultado de nuestro trabajo (puntuación, retroalimentación, fallos cometidos y } \\
\text { puntos a destacar }\end{array}$ \\
\hline 18 & $\begin{array}{l}\text { Los trabajos han sido de alguna manera valorados públicamente ante el resto de la } \\
\text { clase }\end{array}$ \\
\hline 19 & $\begin{array}{l}\text { Las tareas y actividades concretas que cada miembro de los equipos debía realizar } \\
\text { han estado claras desde el inicio tanto en contenido como en plazo (tiempo de } \\
\text { realización) }\end{array}$ \\
\hline 20 & $\begin{array}{l}\text { Tus compañeros han contribuido al equipo tal y como se estableció y ha sido } \\
\text { necesario }\end{array}$ \\
\hline 21 & $\begin{array}{l}\text { Ha existido colaboración entre los miembros del equipo (nos hemos ayudado, } \\
\text { compartido información, comunicado las dificultades etc.) }\end{array}$ \\
\hline 22 & $\begin{array}{c}\text { En su momento cada líder de equipo ha sido aceptado por todos los miembros del } \\
\text { mismo }\end{array}$ \\
\hline 23 & Los líderes han dirigido y coordinado las actividades del equipo \\
\hline
\end{tabular}

\section{ANEXO E. Ítems de Cultura}

\begin{tabular}{|c|c|}
\hline Número & Pregunta \\
\hline 01 & ¿En la universidad donde estudias se fomenta la innovación? \\
\hline 02 & ¿En la universidad donde estudias se promueve el trabajo en equipo? \\
\hline 03 & ¿Para la universidad donde estudias lo más importante es el cumplimiento de las \\
metas?
\end{tabular}

